\title{
RECOMMENDATIONS WIRELESS BROADBAND TECHNOLOGY FOR BACKHAUL RURAL BROADBAND INTERNET SERVICE WITH AFFORDABLE COST
}

\author{
Sabri Balafif ${ }^{1}$, R.V. Hari Ginardi ${ }^{2}$, Febriliyan Samopa ${ }^{3}$ \\ ${ }^{\text {I} S t u d e n t, ~ D e p a r t m e n t ~ o f ~ I n f o r m a t i o n ~ T e c h n o l o g y ~ M a n a g e m e n t ~-~ T e n t h ~ N o v e m b e r ~ I n s t i t u t e ~ o f ~ T e c h n o l o g y ~(I T S) ~}$ \\ ${ }^{2}$ Supervisor, Department of Information Technology Management - Tenth November Institute of Technology (ITS) \\ ${ }^{3}$ Co-Supervisor, Department of Information Technology Management - Tenth November Institute of Technology (ITS)
} sabri.balafif@gmail.com ${ }^{1}$,hari.ginardi@gmail.com²,samopa@gmail.com ${ }^{3}$

\begin{abstract}
Side of the access network has a lot of growing to serve the high-speed internet access such as WiMAX and LTE that is able to deliver access to data in order Megabit per sector. But this technology does not mean anything if the operator is not able to provide the network transport (backhaul) appropriate to support the capacity requirements of the access network. Ideally the appropriate technology to support that issues is the use of technology-based wireless backhaul network E1 / T1, Side of the access network has a lot of growing to serve the high-speed internet access such as WiMAX and LTE that is able to deliver access to data in order Megabit per sector. But this technology does not mean anything if the operator is not able to provide the network transport (backhaul) appropriate to support the capacity requirements of the access network. Ideally the appropriate technology to support above issues is the use of technology-based wireless backhaul network E1 / T1, but the the main constraint is the cost of the use of these technologies are very expensive, because each time a multiple E1 / T1 leased line rental charges mean increased twice. The use of these technologies be suitable if it is implemented in a rural area (non-financially feasible), because the Internet penetration is still below 25\%. One alternative technology capable of solving these challenges is based Wireless Broadband Radio frequency technology. Departing on this view, this research is to measure the maximum performance out of these technologies. This research is descriptive and exploratory because no hypothesis formulated from the beginning to prove. The suitability of the recommended technologies, will test the capacity dimensioning method. From field testing got result that devices from Ubiquiti wireless broadband network that is AirFiber (AF) 24 can be used as a backhaul equipment. It can be concluded based on the ability of AF 24 in transmitting data at 1 Gb (aggregate throughput) at a distance of 2-7 KM in providing high-speed internet access in rural areas even in the condition Near Line Of Sight (NLOS) due to extreme weather.
\end{abstract}

Keyword : Rural Wireless Broadband, Airfiber 24, Hybrid Division Duplexing

$* * *$

\section{INTRODUCTION}

There is a double increasing of Internet traffic over mobile networks which happend in every 12 until 18 months due to consumers trend of using the data packet service. Unlike voice service which is paid by minute of usage (MoU), data packet service rates are based on the volume of data that customers enjoy. Whereas large volumes of data in the data packet service network overload that affects the swelling leased backhaul line as well as in terms of revenue it is not comparable to the increaseing of transport network load that is used for backhaul. A data flat rate schemes (the calculation of cost of Internet access that does not rely on internet access time or the amount of data consumed) seems to be still in use or continue. That means Internet data access will still increase. This led to the phenomenon of what is referred as traffic revenue divergence (as ilustrate in figure 1 ).

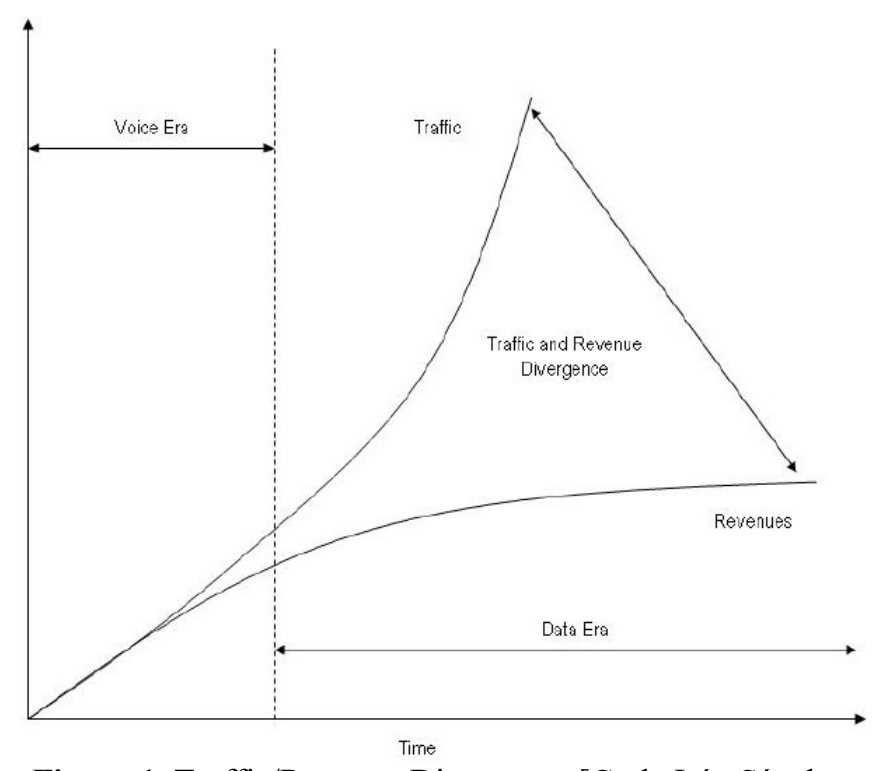

Figure 1. Traffic/Revenue Divergence [Carla Irán Sánchez Aguilar.2016] 
The occurrence of an exponential increase in traffic while the operator revenue remains flat causes wider gap between revenue and cost. Therefore it is important for the operators to create an efficient scheme so that the revenue wont further eroded by its cost. It is by reducing cost in the backhaul network as this cost is the largest component of all.

Rural area with obstacles in accessibility and number of residents becomes factors of consideration for operators of internet service providers in making decision to expand its reach. Providing high speed internet access in rural areas requires infrastructure that connects the access system that is integrated with the network system in city (Global Internet Access), In theory, the ideal media to integrate network system in city with network system in village is a wireless technology E1 / T1. But the costs of this technology is expensive, because everytime E1 / T1 multiplied the leased line rental costs will increase twice. If a mobile operator has $10,0003 \mathrm{G} \mathrm{BTS} /$ base point location, with E1lease up to 15 million per month, while each point requires 3 to 5 times E1, this results OPEX cost becomes very expensive. While the increasing cost becomes main concerns of nowadays Internet service provider operators. Can you imagine if the backhaul transport costs can reach $70 \%$ of the total cost of network management both from capex and opex [Wahyu, A. Ali Muayyadi, Heroe Wijanto.2011].

By considering the above facts and risks, it is necessary to do a feasibility study to get scientific measurable results about the opportunities and potential in building the wireless broadband based internet infrastructure in rural areas by using alternative technology such as Broadband Wireless which use Radio frequency technology.

\section{LITERATUR REVIEW}

\subsection{Backhaul Network}

In a hierarchical telecommunications network the backhaul portion of the network comprises the intermediate links between the core network, or backbone network and the small subnetworks at the "edge" of the entire hierarchical network. ackhaul is the obligation to carry packets to and from that global network. A non-technical business definition of backhaul is the commercial wholesale bandwidth provider who offers quality of service (QOS) guarantees to the retailer. [Wikipedia].

\subsection{Wireless broadband berbasis Radio Frequency}

Signal RF (Radio Frequency) is the electromagnetic waves used by the communication system to send information through the air from one point to another point. Signal RF is also a common means to send data over a wireless network.

Wireless broadband is a wireless connection with large bandwidth of data so it can transmit data at high speed. Broadband or wideband technology is media transmition technology that supports multiple frequencies, ranging from voice to video frequencies. This technology can carry multiple signals by dividing its capacity (which is huge) in some bandwidth channel. Each channel operates on specific frequency. In simple words, Broadband technology is a connection speed of $10 \mathrm{Mbps}$ or more.

The Federal Communications Commission (FCC) defines broadband technology at a minimum speed of $25 \mathrm{Mbps}$ download threshold up to $100 \mathrm{Mbps}$.

\subsection{Innovative Proprietary Modem Technology}

from Ubiquiti Network (Ubiquiti Networks, Inc. 2013-2014)

airFiber ${ }^{\circledR}$ modem technology was purpose-built to address the specific challenges of outdoor, PtP (Point-to-Point) bridging and high-performance network backhauls. airFiber supports FDD (Frequency Division Duplexing), TDD (Time Division Duplexing), and HDD (Hybrid Division Duplexing). FDD operates in full-duplex mode. Typically airFiber achieves the best speed and lowest latency when it is configured as a full-duplex system using FDD. For airFiber in FDD mode, there are two wireless streams: one wireless stream (on one frequency) for TX (Transmit), and one wireless stream (on a different frequency) for $R X$ (Receive). The transmitter and receiver are running concurrently in time. Because of the trade-off between bandwidth resources and propagation conditions, this approach is typically reserved for links in installation sites that have a clear line of sight and are free of reflected energy.TDD operates in halfduplex mode, and TDD links are typically installed in environments that are highly reflective or subject to scattering due to heavy rain or foliage loss. One problem with TDD is propagation delay (amount of time it takes for one transmitted frame to reach the receiving radio). With larger propagation delays, when one radio transmits, the receiving radio is waiting for the transmission to arrive, and there can be times when both radios are in a receiving state. This can cause efficiency issues, reducing throughput and increasing latency. airFiber uses TDD with patent-pending $H D D$ technology to virtually eliminate packet transmission latency. airFiber can transmit data synchronously without any wait time during half-duplex mode, so airFiber's TDD with HDD technology performs significantly better than conventional TDD using less efficient protocols.

\section{Send Packets in Precise Synchronization}

$T X$ and $R X$ frames are completely concurrent and therefore do not consume excessive amounts of overhead, which typically happens in conventional TDD systems. Based on the ranging algorithm built into the air protocol, the airFiber radios use breakthrough HDD technology to calculate the propagation delay and know when each radio can transmit and receive, so they send packets in precise synchronization. The transmissions remain clear even though the packets cross paths half-way. GPS is used to trigger the airFiber radios to transmit at the same time; however, the primary benefit of GPS is that it enables the concurrency of TX and $R X$ frames for co-location of master nodes (airFiber radios).

\section{Increase Throughput and Reduce Latency}

$H D D$ combines the latency performance of FDD with the spectral efficiency of TDD. The airFiber radios virtually 
eliminates wait time (dead airtime) when neither radio is transmitting; instead the transmissions are synchronous. This results in greater efficiency: higher throughput and lower latency.

\subsection{Radio Spectrum Allocation}

To fulfill requirements of internet access services, regulatory has allocated radio frequency spectrum for wireless broadband services. The allocation of the frequency divided by 2 is: a licensed spectrum and unlicensed spectrum, Special use of licensed spectrum must have licenses from Federation Communication Commission (FCC) and the regulatory responsibility for the radio spectrum each country. While for the unlicensed spectrum is not necessary for frequency licenses to use it but must use certified radio equipment. Radio frequency spectrum is unlicensed classified:

- $\quad$ ISM-2.4 pada $2.4-2.4835 \mathrm{GHz}$

- ISM-5.8 pada 5.725-5.850 GHz

- U-NII - $5.15-5.35 \mathrm{GHz}$

- U-NII- 5.725-5.826 GHz

\section{RESEARCH METHOD}

This research is descriptive and exploratory because no hypothesis to prove is formulated from the beginning. The recommended technology will be tested by using the capacity dimentioning method approach.

\section{ANALYSIS}

\subsection{Challenge}

Challenges in the use of wireless technology (Technical Issue) for providing internet broadband in rural is Extrem weather conditions (heavy rain and thick fog) cause the track of radio waves to be in category of near line of sight (NLOS)

\subsection{Testing Scenario Devices}

To achieve Flexibility in Backhaul Network, The topologies will use recommendation from ubiquiti Network. Simple Point to Point (PtP) link design used in ring redundancy and daisy-chained paths.

\subsubsection{Scenario Ring Redundancy}

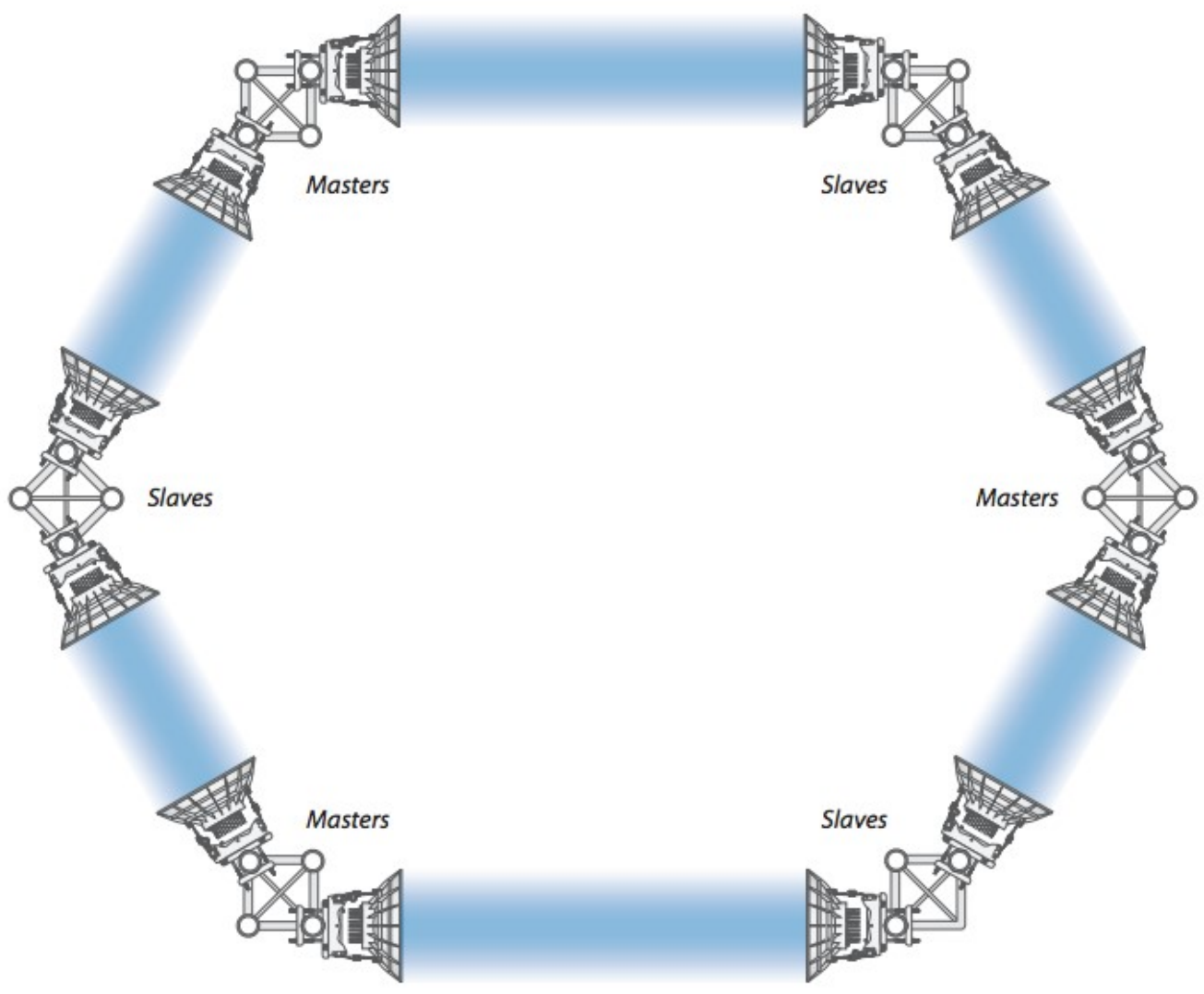

Figure 2. Ring Redundancy Topology

Multiple AF24 radios can be placed in a ring-topology for redundancy. If one link goes down, other links have alternate routes available. For each link, configure one airFiber radio as Master, and configure the other as Slave. 


\subsubsection{Scenario Daisy-Chain Paths}

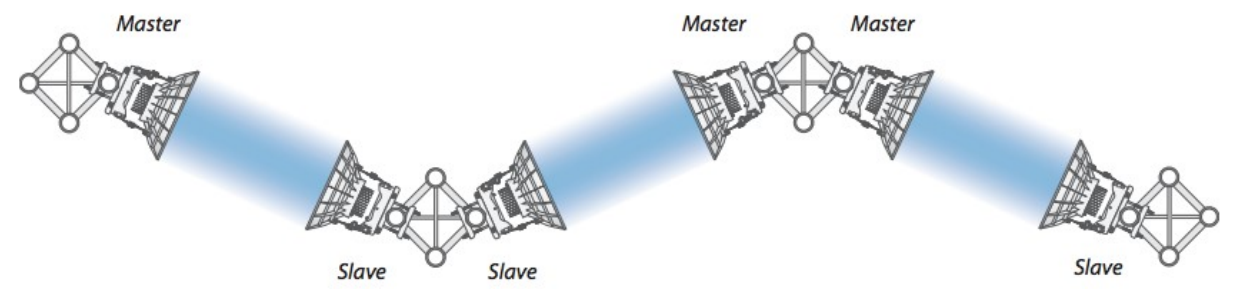

Figure 3. Daisy-Chain Paths Topology

Multiple AF24 radios can also be used to extend the distance of a link like a series of PtP relays (PtPtP). Radios in the same node must use the same Wireless Mode (Master or Slave) to ensure low-latency across the entire link.

1. Device Architecture AirFiber 24:

\begin{tabular}{|c|c|c|c|}
\hline Information & \multicolumn{3}{|c|}{ Key Architecture } \\
\hline Features & \multicolumn{3}{|c|}{$\begin{array}{l}\text { - Hybrid Division Duplexing (HDD) } \\
\text { - Dual-independent Antenna Architecture (simultaneous transmission and reception in the same time) } \\
\text { - GPS Synchronization } \\
\text { - Adaptive Modulation and Coding }\end{array}$} \\
\hline Engine & \multicolumn{3}{|c|}{ INVICTUS ${ }^{\mathrm{TM}}$ (Proprietary Technology from Ubiquiti Network) } \\
\hline Frequency & \multicolumn{3}{|c|}{$24 \mathrm{Ghz}$} \\
\hline Max. Modulation & \multicolumn{3}{|c|}{ 64QAM } \\
\hline \multirow{2}{*}{ Integrated Split Antenna } & \multicolumn{3}{|c|}{ TX Gain $33 \mathrm{dBi}$} \\
\hline & \multicolumn{3}{|c|}{ RX Gain $38 \mathrm{dBi}$} \\
\hline Beamwidth & \multicolumn{3}{|c|}{$<3.5^{\circ}$} \\
\hline Energy Con. & \multicolumn{3}{|c|}{$50 \mathrm{~W}$} \\
\hline \multicolumn{4}{|c|}{ Estimated rainfall attenuation rates } \\
\hline Distance $(\mathrm{Km})$ & $1.25 \mathrm{~mm} / \mathrm{hr}$ & $5 \mathrm{~mm} / \mathrm{hr}$ & $25 \mathrm{~mm} / \mathrm{hr}$ \\
\hline 1 & $0.2 \mathrm{db}$ & $0.7 \mathrm{db}$ & $4 \mathrm{db}$ \\
\hline 2 & $0.4 \mathrm{db}$ & $1.4 \mathrm{db}$ & $8 \mathrm{db}$ \\
\hline 3 & $0.6 \mathrm{db}$ & $2.1 \mathrm{db}$ & $12 \mathrm{db}$ \\
\hline 4 & $0.8 \mathrm{db}$ & $2.8 \mathrm{db}$ & $16 \mathrm{db}$ \\
\hline
\end{tabular}

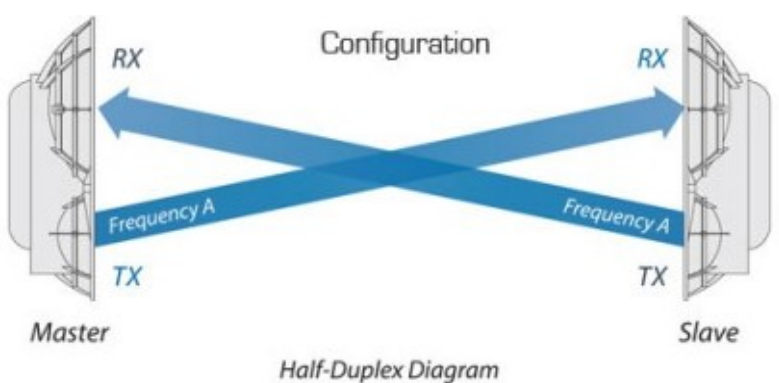

Full Duplex The $T X$ and $R X$ Frequencies should be different.

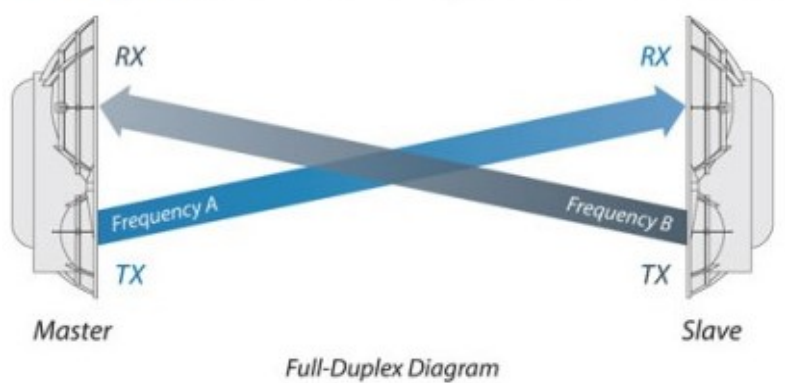

Figure 4. airFiber AF24 Configuration

Based on wireless theory, if the technology could be implemented in real then the throughput provided by using the technology will be up to $1500 \mathrm{Mbps}$ (throughput and range values may vary depending on the environmental condition.

\section{RECOMMENDATION \\ FROM UBIQUITI NETWORK FOR OPTIMIZATION}

\section{\# airFiber - Best location for a high performance link}

\section{Best location}

For best performance, you must install the AF5 as high up as possible, with a line of sight free from obstructions. This is especially true in FDD (Frequency Division Duplexing) mode, as there is a requirement to maximize channel/spectrum reuse. Any objects in front of or near the front of the AF5 can have a significant impact on overall performance. The AF5 should be installed $1 \mathrm{~m}$ (3.3 ft) below the highest point of the structure to reduce the risk of a lightning strike. When the AF5 must be installed above a metallic surface like a corrugated flat roof or water tower, then you should ensure that the AF5 is located more than 3 $m$ (10 feet) above the metal/reflective surface. 


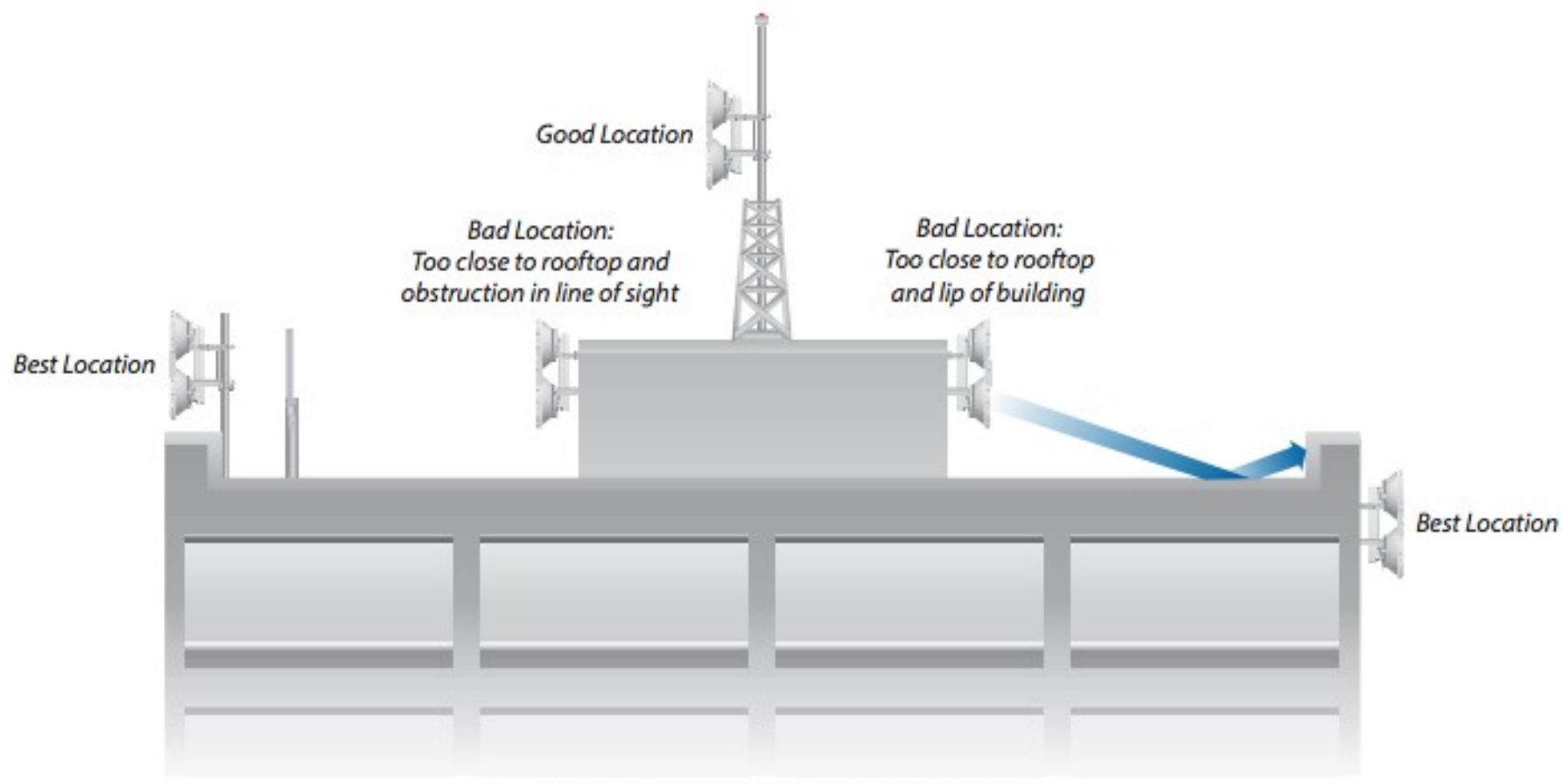

\section{Deployment on Rooftop of Building}

Figure 5. Deployment AF 24

What is generally true for cellular installations is true for the AF5. You should mount the AF5 where it can see no reflections in the near field, so we recommend the following types of mounting sites:

- Perimeter mounting on a water tower/structure (best)

- Mounting near the edge of the top of a structure

- Mounting on an elevated mast or tower

Radios that are mounted anywhere near the surface of a roof or the top of a water tower can be significantly affected by reflections. FDD is more susceptible to the elements of a deployment environment than TDD (Time Division Duplexing) is.

\section{* airFiber - How many radios can I co-locate at the same} location?

Although every scenario will vary in number, Ubiquiti's airFiber radios have a number of design characteristics that make it ideal for co-location backhaul deployments. Listed below are tips for co-locating AF radios:

- In most cases, co-location interference is not a concern because the beamwidth is so narrow (less than 3 degrees). However, to the extent that greater separation (both vertical and lateral) exists between co-located radios, neighbors are less likely to hear each other. About 3-5m of separation is good, but $5 m+$ separation is optimal.

- Co-located AF24 radios are meant to be pointed in different directions to achieve isolation of signals. It is discouraged to point two co-located AF24 radios in the same direction, since this may confuse receiving AF24 radios.

- Co-located AF24 radios must use the same Wireless Mode (Master or Slave). It's discouraged to mix Master and Slave radios at the same tower site.

- Back-to-back AF24 radios can use the same frequency. This is due to the excellent Front-to-Back ratio of the antennas $(\sim 70 \mathrm{~dB})$.

- Different frequencies are generally recommended for adjacent airFiber radios (e.g., top / bottom, side / side), however, this is not a strict requirement.

- Ubiquiti's HDD mode combined with the GPS Sync technology allows all co-located radios to simultaneously transmit. This is extremely effective in minimizing co-location interference.

By applying these tips, we've seen cases of users successfully co-locating as many as 8 AF24 radios on the same tower. Due to numerous differences in link characteristics such as tower height or RF environment, there's no hard / fast rule for the total number of co-located radios. 


\section{RESULT}

The budget calculation link to $5 \mathrm{~km}$ distance as follows:

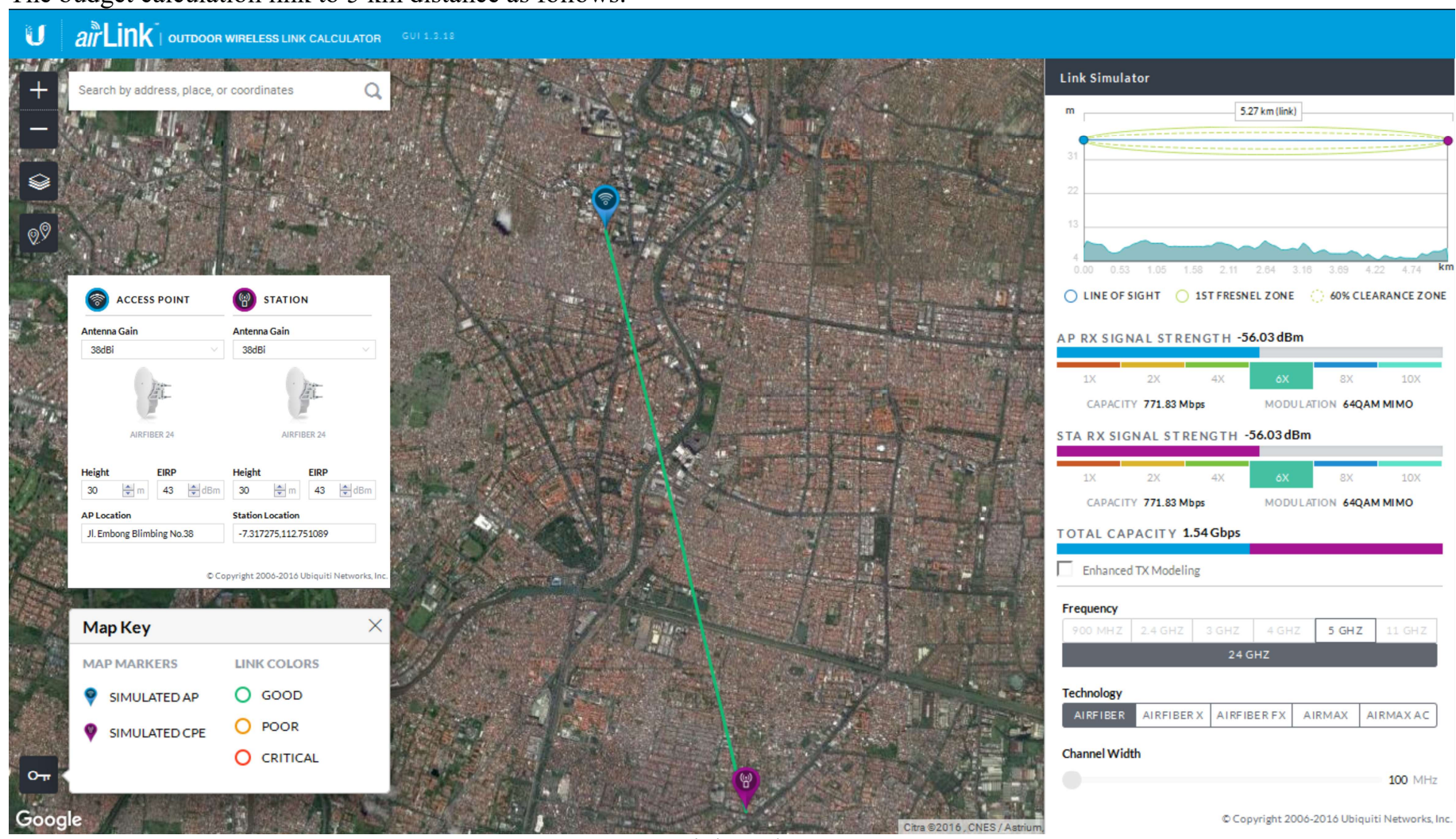

Figure 6. Link Budget AF 24.

The calculation of Link Budget, If the wireless link qualified to achieve $60 \%$ Fresnel zone so AF24 can generate signal signal strength - EIRP of $50 \mathrm{dBm}$ with $43 \mathrm{dBM}$, it will get the results of aggregate throughput of 1.5 Gbps. On realization is obtained which is not far from the link budget calculation, namely: AF24 can generate signal strength - 67
$\mathrm{dBm}$, where the modulation rate is already reached at the highest modulation of the ability of these devices is $6 \mathrm{X}$ 64QAM MIMO. So as to produce aggregate throughput of 1.5 GB with the distribution capacity of the TX (Upload) amounted to $746 \mathrm{Mbps}$ and RX (Download) $770 \mathrm{Mbps}$

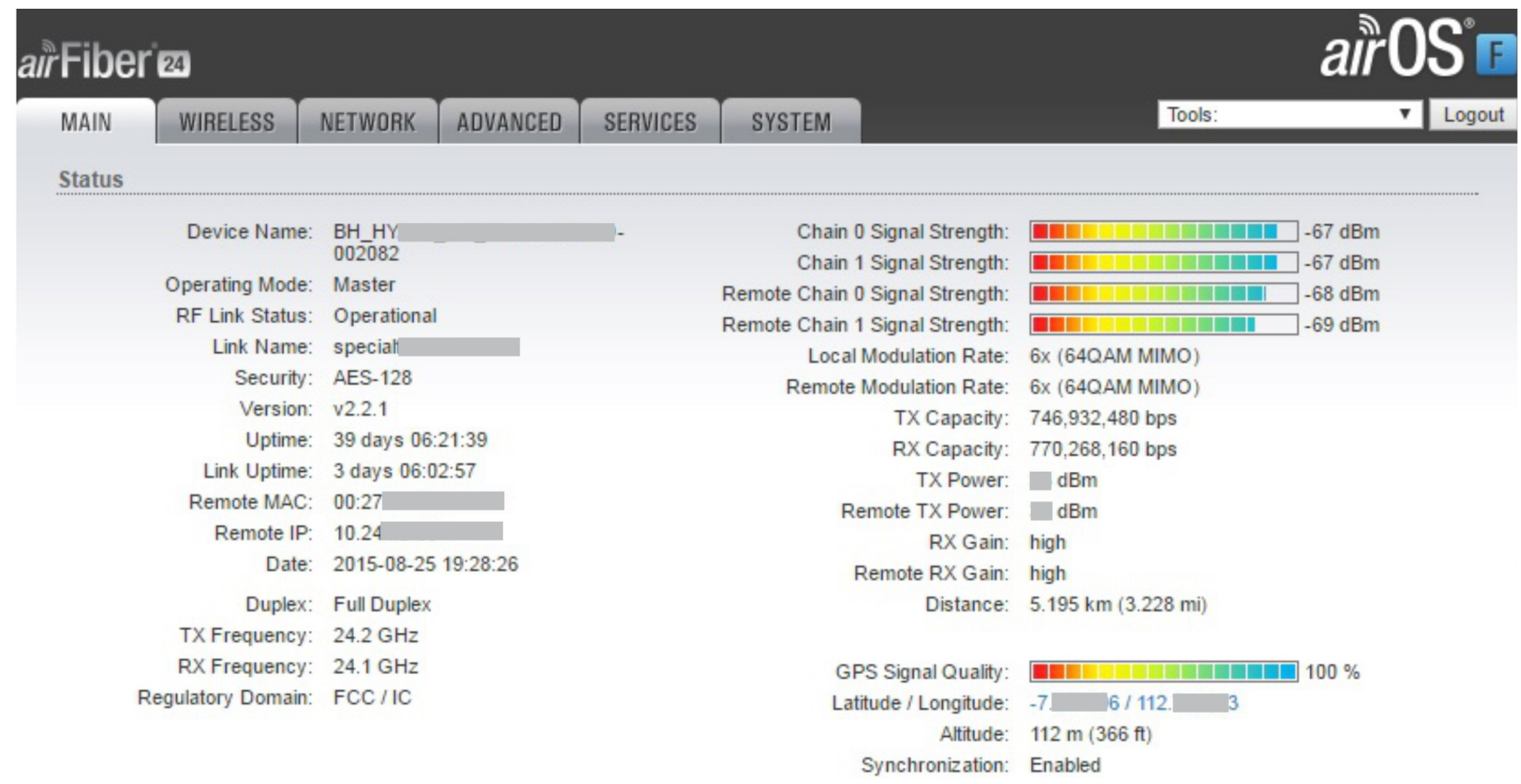

Figure 7. Result Tes AF24 


\begin{tabular}{|c|c|c|c|}
\hline \multicolumn{4}{|c|}{ airFiber AF-24 Receive Sensitivity } \\
\hline Modulation & Sensitivity & FDD Capacity* & TDD Capacity" \\
\hline 64OAM & $-66 \mathrm{dBm}$ & $1500 \mathrm{Mbps}$ & $760 \mathrm{Mbps}$ \\
\hline 16QAM & $-72 \mathrm{dBm}$ & $1000 \mathrm{Mbps}$ & $507 \mathrm{Mbps}$ \\
\hline QPSK MIMO & $-78 \mathrm{dBm}$ & $500 \mathrm{Mbps}$ & $253 \mathrm{Mbps}$ \\
\hline QPSK SISO & $-80 \mathrm{dBm}$ & $250 \mathrm{Mbps}$ & $127 \mathrm{Mbps}$ \\
\hline $1 / 4 \times$ QPSK SISO & $-87 \mathrm{dBm}$ & $62.5 \mathrm{Mbps}$ & 31.7 Mbps \\
\hline
\end{tabular}

Figure 8. AF24 Receive Sensitivity

\section{COST COMPARATION}

\begin{tabular}{|l|c|}
\hline \multicolumn{2}{|c|}{ CAPEX } \\
\hline \multicolumn{1}{|c|}{ Information } & OPEX \\
\hline MSRP/Peer & $1,497.50$ \\
\hline & AirFiber 24 \\
\hline Information & $\$ 8000 /$ Year \\
\hline Monitoring frequency & $\$ 1800 /$ Year \\
\hline \multirow{2}{*}{ Licensed Bands } & depend country regulation \\
\cline { 2 - 3 } & Free because Handle by Distributor \\
\hline Licensed Radio & $50 \mathrm{~W}$ \\
\hline
\end{tabular}

\section{CONCLUSION}

To achieve the Network Flexibility in NLOS due Extrem weather (heavy rain and thick fog), can be done by establishing a backhaul network using protocols Hybrid Division Duplexing (HDD) with the Engineering Adaptive Modulation and Coding (AMC). It is intended that wireless devices can adjust their performance by choosing the type of modulation and coding accordance with channel conditions to obtain the best performance of Bit Error Rate (BER) value

\section{REFERENCES}

[1] Ubiquiti Networks, Inc. HDD (Hybrid Division Duplexing), 2014

[2] FCC "Part 15: Radio Frequency Devices", Federal Communications Commission. (2001)

[3] Carla Irán Sánchez Aguilar.2016White Paper The relevance and critical success factors of Project Management in the face of convergence - Scientific Figure on ResearchGate. Available from: https://www.researchgate.net/265231121_fig1_Figure1-TrafficRevenue-Divergence [accessed 26 Jan, 2017]

[4] Wahyu, A. Ali Muayyadi, Heroe Wijanto.2011.NALISIS KEBUTUHAN BANDWIDTH BACKHAUL SELULER PADA OPERATOR $X$. Magister Elektro Komunikasi, Fakultas Teknik Elektro, Universitas Telkom 